\title{
Interweave Cognitive Networks with Co-operative Sensing
}

\author{
S. Kusaladharma and C. Tellambura, Fellow, IEEE \\ Department of Electrical and Computer Engineering \\ University of Alberta, Edmonton, Alberta T6G 2V4, Canada \\ Email: kusaladh@ualberta.ca and chintha@ece.ualberta.ca
}

\begin{abstract}
This paper investigates the effects of different cooperative sensing strategies on erroneous spectrum sensing for an interweave cognitive radio network. The setup is as follows. Primary receiver nodes and secondary nodes are randomly distributed in $\mathbb{R}^{2}$. We model them as two independent homogeneous Poisson point processes. Beacon (out-of-band) signals, periodically transmitted by primary receivers, indicate to the secondary nodes that spectrum is occupied. Whenever beacon detection fails, the transmissions of secondary nodes generate harmful interference. Thus, to alleviate this issue, the misdetection probability of secondary nodes must be reduced. To this end, we propose two strategies: 1) a secondary node cooperates with its closest neighbour, and 2) a secondary node cooperates with $M$ random neighbours within a given radius. Furthermore, along with these co-operation strategies, we investigate three primary beacon detection methods for secondary nodes: 1) separately decoding each primary beacon, 2) detecting only the closest primary receiver's beacon, and 3) detecting the aggregate beacon signal from all primary receivers. For the exponential path loss and Rayleigh fading considered, we derive the total misdetection probability for each scheme along with the resulting outage probability of a primary receiver. We finally show through numerical results that $M$ co-operation works better for lower reception thresholds, and that for a reception threshold of $-120 \mathbf{d B m}, \mathbf{a} 10^{4}$ fold decrease in the misdetection probability is achievable.
\end{abstract}

\section{INTRODUCTION}

Interweave cognitive radio (CR) networks enable secondary users to opportunistically access unused primary primary user spectrum [1], and beacon signalling by primary user devices has received attention as a means to indicate spectrum occupation [2], [3]. The secondary nodes refrain from transmitting whenever they detect a beacon. However, beacon misdetection is a significant potential problem. Whenever this occurs, the resultant transmissions from the misdetecting secondary users create interference.

Incorrect spectrum sensing can be mitigated by allowing secondary nodes to share their decisions [4]. Termed cooperative spectrum sensing, this process attempts to negate beacon misdetection when the fading and shadowing are uncorrelated [5]. However, co-operation requires additional resources on the part of the secondary nodes, and faces several implementation challenges. The number of co-operating nodes and their locations are important parameters to consider when assessing the effectiveness of secondary user co-operation [6]. Moreover, due to the random nature of node deployments, path loss, and fading, the shared spectrum information itself may be corrupted. As such, characterizing the misdetection probability of a secondary node employing co-operation and the resulting primary user interference is essential to further develop CR standards.

\section{A. Prior research}

Several previous works investigate interference issues in interweave cognitive radio networks due erroneous spectrum sensing, while others incorporate co-operative spectrum sensing. A statistical model for interference in spectrum sensing cognitive networks is developed in [2] and [7] while [8] analyzes the primary users' coverage probability under erroneous sensing and false alarms. Statistics of aggregate secondary interference are obtained in [9] by incorporating spectrum sensing and power control. Moreover, [10] derives the moment generating function and the expectation of the aggregate interference for a spectrum sensing CR network. Reference [11] analyzes the geometric region allowing CR transmission with the help of co-operative sensors. Furthermore, [12] develops statistical models for the interference distribution and closed-form expressions for the capacityoutage probability, while [13] develops models for bounding interference levels using a modified Matern process for the secondary nodes. An analytical framework for the analysis and design of co-operating spectrum sensing methods over correlated shadow fading environments is proposed in [14].

\section{B. Motivation and contribution}

Most previous works incorporating co-operative spectrum sensing for a CR network have either assumed a constant number of secondary nodes, a single primary receiver, or perfect dissemination of sensed spectrum information by the co-operating nodes. However, in reality, the number and locations of secondary nodes are random. Furthermore, a single transmitter-receiver pair for the primary system is unrealistic since multiple primary receivers may operate within any given area. As such, both primary receivers and secondary nodes need to be modeled using a stochastic process. In addition, information shared by the co-operating nodes themselves will be affected by the channel and spatial conditions, and thus may be misdetected as well.

Therefore, to this end, we will model the primary receivers and the secondary nodes as Poisson Point Processes (PPPs) [15], and consider a channel having exponential path loss and Rayleigh fading. Each primary receiver sends out a beacon signal whenever they are active. We will consider three different schemes for primary beacon detection: 1) aggregate beacon power, 2) separately sensing the beacon from each primary 
receiver, and 3) detecting the beacon of the primary receiver providing the best average received signal (i.e. the closest). If a primary beacon is sensed, the co-operating secondary nodes send another beacon signal. Both primary and secondary node beacons may be misdetected due to channel conditions. For co-operation, we consider two cases where each secondary receiver co-operates 1) with its closest neighbor and 2) with $M$ randomly selected secondary nodes located within a given radius. An OR scheme is used to combine the received primary and secondary information. We analyze the total misdetection probability under the aforementioned schemes and investigate the effectiveness of co-operative sensing under different system parameters. Moreover, we derive the outage probability of a primary receiver to characterize its performance.

Notations: $\Gamma(x, a)=\int_{a}^{\infty} t^{x-1} e^{-t} d t$ and $\Gamma(x)=\Gamma(x, 0)$ [16]. $\operatorname{Pr}[A]$ is the probability of event $A, f_{X}(\cdot)$ is the probability density function $(\mathrm{PDF}), F_{X}(\cdot)$ is the cumulative distribution function $(\mathrm{CDF}), M_{X}(\cdot)$ is the MGF, and $E_{X}[\cdot]$ denotes the expectation over random variable $X$.

\section{A. Spatial distribution}

\section{SYSTEM MODEL}

We consider a system of primary and cognitive nodes located in $\mathbb{R}^{2}$. One primary transmitter serves a set of primary receivers, where the receiver number and locations are random. Such a setup is common in terrestrial television systems for which CR techniques have been envisioned. Within the same geographical area, there also exists a secondary network which utilizes spectrum holes in the primary network to transmit data. Such a network would practically be an ad-hoc network or a sensor network. The secondary network's nodes are also randomly located.

Modeling random node distributions through PPPs has become popular in the literature [7]. Ideally, the secondary nodes would form a Poisson cluster process where the different clusters represent different secondary networks. However, further analysis with Poisson cluster processes is difficult. As such, we model the primary receivers and the secondary nodes as two independent homogeneous PPPs. Let $\Phi_{p}$ and $\Phi_{s}$ be the processes of the primary receivers and the secondary nodes with respective intensities $\lambda_{p}$ and $\lambda_{s}$. Because homogeneous PPPs are considered, $\lambda_{p}$ and $\lambda_{r}$ are constant, and do not depend on the spatial location. Thus $\Phi_{p}$ or $\Phi_{s}$ is a point processes in $\mathbb{R}^{2}$ with uniform intensity $\lambda>0\left(\lambda \in\left\{\lambda_{p}, \lambda_{s}\right\}\right)$ such that: (a) for every bounded closed set $A$, the number $N(A)$ is Poisson distributed with

$$
\operatorname{Pr}[N(A)=n]=\frac{(\lambda A)^{n}}{n !} e^{-\lambda A},
$$

and (b) if $A_{1}, A_{2} \ldots, A_{m}$ are disjoint sets, $N\left(A_{1}\right), N\left(A_{2}\right), \ldots, N\left(A_{m}\right)$ are independent random variables [17].

We will assume that there is no channel state information (CSI) available at secondary nodes about the primarysecondary and secondary-secondary links. This assumption is indeed reasonable and practical for a cognitive ad-hoc network. Moreover, mobility of secondary and primary nodes will not be considered in this analysis. Practically, primary receivers such as digital television subscribers will be stationary. Moreover, even if the secondary users move randomly (e.g. random walk or the Brownian motion), a snapshot of the network at any time $T_{i}$ would still generate a homogeneous PPP. Furthermore, we consider the secondary nodes to be always ready to transmit data whenever a spectrum white space is detected, and that all the primary receivers are active. There is no loss of generality in these assumptions since activity factors can easily be incorporated using the Coloring Theorem [15]. This theorem says that if nodes of a PPP $\Phi$ with intensity $\lambda$ is marked independently, and $p_{t}$ is the probability of a node receiving the $t$-th color, the set of nodes with the $t$-th color forms a PPP $\Phi_{t}$ with intensity $p_{t} \lambda$. Thus, if a primary receiver is active with an activity factor of $q_{p}$, the set of active primary receivers follow a thinned PPP with intensity $q_{p} \lambda_{p}$. The same argument holds for the secondary nodes.

\section{B. Signal propagation}

The signals undergo Rayleigh fading and exponential path loss. We assume that the fading is uncorrelated between different nodes. With Rayleigh fading, the channel power gain of the $i$-th channel $|h|^{2}$ follows the exponential distribution with density $f_{|h|^{2}}(x)=e^{-x}, 0<x<\infty$. The average received power at a receiver at a distance $r$ from the transmitter $P_{R}$ is denoted as $P_{R}=C P g(r)$, where $P$ is the transmit power level of the device, $A$ is a constant depending on the frequency and antennas, and $g(r)$ is the distance dependent path loss. For simplicity, we will use $C=1$ from here on in. If the path loss exponent is $\alpha, g(r)=\min \left(1, r^{-\alpha}\right)$. In effect, whenever $r>1$, the received power follows the simplified path loss model of [18] $P_{R}=\mathrm{Pr}^{-\alpha}$.

We assume that all secondary users transmit at a fixed power level. Power control and receiver association procedures by the secondary users will not be considered as it is out of the scope of this paper.

\section{Spectrum sensing}

While spectrum sensing techniques include energy detection, cyclostationary detection, and matched filter detection [4], out-of-band sensing is a viable approach [3]. Out-ofband sensing utilizes beacon signals transmitted periodically on a control channel by the primary system devices [3], [19], and the secondary devices opportunistically access the spectrum whenever the beacon signal is not present. While both primary transmitters and receivers can transmit a beacon, it is more advantageous to have it transmitted by the receivers as those are the devices affected from concurrent secondary transmissions.

In our system, primary receivers transmit a low powered beacon signal whenever they are active. We consider three models for the reception of primary receivers' beacon signals by secondary nodes. They are: 1) aggregating beacon power from all primary receivers in range, 2) sensing each primary beacon separately and using an OR rule, and 3) sensing the beacon from the closest primary receiver (the primary receiver having the highest average signal power). 
The secondary nodes co-operate with each other during the beacon reception to improve the chances of correct reception. However, if a sensing error is made, the resulting secondary transmissions cause interference and performance degradation to the primary users. Secondary nodes may employ decision fusion and data fusion [4] to co-operate. Decision fusion involves each cooperating secondary node making a spectrum decision before transmitting that decision whereas with data fusion, each secondary node simply amplifies and transmits the received beacon. In our analysis, we will consider decision fusion. Moreover, two cases are considered for selecting cooperating nodes: 1) co-operating with the nearest secondary node, and 2) co-operating with $N$ nodes within a radius of $R_{c}$.

It is assumed that the secondary nodes have the ability to differentiate the beacon signals from the primary receivers from the spectrum information coming from other cooperating secondary nodes. This can be achieved by techniques such as using separate orthogonal codes for different secondary nodes and primary receivers, using different time slots, or having a separate narrow band channel for secondary user spectrum information sharing.

\section{PRIMARY BEACON MisdeteCtion}

In CR networks where the primary user performance must be guaranteed, the missed detection probability remains the most vital performance measure. This section provides an analysis of the primary beacon missed detection probability before co-operation takes place.

\section{A. Aggregating beacon power}

We will first look at a scheme where each secondary node senses the spectrum based on the aggregate beacon power received. This scheme assumes that the secondary nodes cannot differentiate among the different primary receiver beacons.

Let the $i$-th secondary node located at $x_{i}$ be denoted as $\phi_{s, i} \in \Phi_{s}$, and the $j$-th primary receiver located at $y_{j}$ be denoted as $\phi_{p, j} \in \Phi_{p}$. If $r_{i, j}$ is the distance from $\phi_{s, i}$ and $\phi_{p, j}, r_{i, j}=\left\|x_{i}-y_{j}\right\|$. However, as $r_{i, j}$ becomes large, the path loss $g\left(r_{i, j}\right) \rightarrow 0$. As such, the received beacon power from $\phi_{p, j}, \forall r_{i, j}>R_{e}$ is considered to be 0 , where $R_{e}$ is an outer distance. Due to $\Phi_{p}$ being a homogeneous PPP, the CDF of $r_{i, j}$ can be obtained using the average number of nodes within a fixed area. Thus, $F_{r_{i, j}}(x)=\frac{x^{2}}{R_{e}^{2}}, 0<x<R_{e}$, The PDF is obtained through the differentiation of the CDF as

$$
f_{r_{i, j}}(x)=\left\{\begin{array}{rl}
2 \frac{x}{R_{e}^{2}} & , 0<x<R_{e} \\
0 & , \text { otherwise }
\end{array} .\right.
$$

All $\phi_{p, j} \in \Phi_{p}$ transmit a constant low powered beacon signal. Let $P_{b}$ denote its transmit power level. If $P_{R}$ is the received beacon power at secondary node $\phi_{s, i} \in \Phi_{s}$,

$$
P_{R}=\sum_{j \in \Phi_{p}} P_{R, j}
$$

where $P_{R, j}$ is the received beacon power from $\phi_{p, j}$. We can write $P_{R, j}$ as $P_{R, j}=P_{b}\left|h_{i, j}\right|^{2} g\left(r_{i, j}\right)$. The received signal to noise ratio $\gamma$ of the beacon signal at $\phi_{s, i}$ becomes $\gamma=\frac{P_{R}}{\sigma_{b}^{2}}$, where $\sigma_{b}^{2}$ is the noise power spectral density of the beacon channel. The secondary nodes can employ energy detection of the beacon channel or use a received power threshold in order to ascertain a beacon's presence. However, as shown in [12], even an energy detection based scheme can be approximated as a simple received power threshold based scheme with an appropriate threshold. Therefore, in our analysis, a detection occurs whenever $P_{R}<P_{t h}$, where $P_{t h}$ is the reception threshold.

Let $q_{p, i}$ be the probability of primary beacon misdetection by $\phi_{s, i}$ before any co-operation takes place. $q_{p, i}$ is written as

$$
q_{p, i}=\operatorname{Pr}\left[P_{R}<P_{t h}\right]=F_{P_{R}}\left(P_{t h}\right),
$$

which is the CDF of $P_{R}$. This can be evaluated using an MGF based approach. Let $M_{P_{R}}(s)$ be the MGF of the received beacon power at $\phi_{s, i}$. From the definition of the MGF, $M_{P_{R}}(s)=E\left[e^{-s P_{R}}\right]$. If $M_{P_{R, j}}(s)$ is the MGF of the received beacon power from $\phi_{p, j}$, and $N$ is a Poisson random variable with a distribution given by (1),

$$
M_{P_{R}}(s)=E_{N}\left[\left(M_{P_{R, j}}(s)\right)^{N}\right]=e^{\pi R_{e}^{2} \lambda_{p}\left(M_{P_{R, j}}(s)-1\right)} .
$$

$M_{P_{R, j}}(s)$ is obtained as follows.

$$
\begin{aligned}
& M_{P_{R, j}}(s)=E\left[e^{-s P_{b}\left|h_{i, j}\right|^{2} g\left(r_{i, j}\right)}\right] \\
= & \int_{0}^{1} \frac{1}{1+s P_{b}} \frac{2 r_{i, j}}{R_{e}^{2}} d r_{i, j}+\int_{1}^{R_{e}} \frac{1}{1+s P_{b} r_{i, j}^{-\alpha}} \frac{2 r_{i, j}}{R_{e}^{2}} d r_{i, j}(5)
\end{aligned}
$$

A closed form expression for the second integral is not apparent. Therefore, as an approximate simplification using the series summation $(1+x)^{-1}=\sum_{k=0}^{\infty}(-x)^{k}$, we obtain a simplified expression for $M_{P_{R, j}}(s)$ as

$$
M_{P_{R, j}}(s)=\frac{1}{R_{e}^{2}}\left(\frac{1}{1+s P_{b}}+\sum_{l=0}^{\infty} 2\left(-s P_{b}\right)^{l} \frac{R_{e}^{2-\alpha l}-1}{2-\alpha l}\right) .
$$

$F_{P_{R}}(x)$ can be obtained through the inverse Laplace transform by $F_{P_{R}}(x)=\mathcal{L}^{-1}\left(\frac{M_{P_{R}}(s)}{s}\right)$, and replacing $x$ with $P_{t h}$ gives $q_{p, i}$.

Aggregating beacon power will invariably give the best results in terms of the average misdetection probability. However, conversely, this scheme is not entirely suitable for a CR network among densely deployed primary receivers. This is because CR nodes will hardly get any chance to conduct their transmissions.

\section{B. Separately sensing primary beacons}

For this scheme, we assume that the secondary nodes can differentiate the beacons coming from various primary receivers (practically done by using different orthogonal codes for each primary receiver). Furthermore, a secondary node senses each beacon separately, and uses an OR rule to decide on whether a primary beacon is present. In other terms, even if the beacon from one primary receiver is detected, no misdetection would occur. The downside of this scheme is that it quickly becomes unpractical when $\lambda_{p}$ increases. 
$q_{p, i}$ for this scheme is written as $q_{p, i}=\left(\operatorname{Pr}\left[P_{R, j}<P_{t h}\right]\right)^{N}$, where $P_{R, j}$ is the beacon power from $\phi_{p, j}$ received at $\phi_{s, i}$ defined in the previous section, and $N$ is a Poisson random variable following the distribution of (1). $\operatorname{Pr}\left[P_{R, j}<P_{t h}\right]$ is written as $\operatorname{Pr}\left[P_{R, j}<P_{t h}\right]=E_{r_{i, j}}\left[1-e^{-\frac{P_{t h}}{P_{b} g\left(r_{i, j}\right)}}\right]$. Thus, $q_{p, i}$ becomes

$$
e^{-\pi R_{e}^{2} \lambda_{p}\left(\frac{e^{-\frac{P_{t h}}{P_{b}}}}{R_{e}^{2}}+\frac{2}{R_{e}^{2}} \int_{1}^{R_{e}} e^{-\frac{P_{t h}}{P_{b} r_{i, j}^{-\alpha}}} r_{i, j} d r_{i, j}\right)} .
$$

Because a closed form solution is not apparent, we can use a numerical technique to evaluate this. Under certain parameter values, a series summation based simplification can be used to simplify (7) which results in

$$
\begin{aligned}
& \text { to simplify (7) which results in } \\
& q_{p, i}=e^{-\pi R_{e}^{2} \lambda_{p}}\left(\frac{e^{-\frac{P_{h}}{P_{b}}}}{R_{e}^{2}}+\frac{2}{R_{e}^{2}} \sum_{k=0}^{\infty} \frac{\left(-\frac{P_{t h}}{P_{b}}\right)^{k}}{k !}\left(\frac{R_{e}^{2+\alpha k}-1}{2+\alpha k}\right)\right) .
\end{aligned}
$$

However, more resources are required for separate sensing, and is invariably more complex. Furthermore, the primary receivers need to be co-ordinated to send separately identifiable beacons. This may not be practical for certain primary receiver types such as digital terrestrial television subscribers.

\section{Closest primary receiver selection}

Within this scheme, each secondary node $\phi_{s, i}$ senses the beacon of the primary receiver closest to it. The closest primary receiver can be found in practice by measuring the average received signal power. Moreover, this scheme assumes that $\phi_{s, i}$ can differentiate between the beacons from different primary receivers.

Let $\phi_{p, 1 \backslash i} \in \Phi_{p}$ be the nearest primary receiver to $\phi_{s, i}$. We assume that $\phi_{p, 1 \backslash i}$ is located at $y_{1 \backslash i} \in \mathbb{R}^{2}$, and that $r_{p, 1}$ is the distance from $\phi_{s, i}$ to $\phi_{p, 1 \backslash i} . r_{p, 1}$ can be written as $\left\|y_{1 \backslash i}-x_{i}\right\|$, and its distribution is obtained using the void probability of a PPP as [20], [21]

$$
f_{r_{p, 1}}(x)=2 \pi \lambda_{p} x e^{-\pi \lambda_{p} x^{2}}, 0<x<\infty .
$$

However, as the signals from $\phi_{p, 1 \backslash i}$ with $r_{p, 1}<R_{e}$ are neglected due to path loss, there may be an occasion where a $\phi_{p, 1 \backslash i}$ does not exist. If this probability is $p_{0}, p_{0}=e^{-\pi \lambda_{p} R_{e}^{2}}$. Whenever this situation occurs, the misdetection probability of $\phi_{s, i}$ will always be 1 . However, conversely, because of high the path in such a scenario, the interfering signals will also have a negligible effect on the primary system. Let $r_{p, 1 \backslash i}$ be the distance from $\phi_{s, i}$ to $\phi_{p, 1 \backslash i}$ whenever $r_{p, 1}<R_{e}$. The PDF of $r_{p, 1 \backslash i}$ is obtained as $f_{r_{p, 1 \backslash i}}(x)=\frac{2 \pi \lambda_{p} x}{1-e^{-\pi \lambda_{p} R_{e}^{2}}} e^{-\pi \lambda_{p} x^{2}}$, $0<x<R_{e}$.

Let $\left|h_{j, 1 \backslash i}\right|^{2}$ be the channel power gain between $\phi_{s, i}$ and $\phi_{p, 1 \backslash i}$. Therefore, when $\phi_{p, 1 \backslash i}$ exists, the received beacon power $\left(R_{b}\right)$ at $\phi_{s, i}$ from $\phi_{p, 1 \backslash i}$ is given by $R_{b}=$ $P_{b}\left|h_{j, 1 \backslash i}\right|^{2} g\left(r_{p, 1 \backslash i}\right)$, where $g\left(r_{p, 1 \backslash i}\right)$ is the path loss between $\phi_{s, i}$ and $\phi_{p, 1 \backslash i}$.

$q_{p, i}$ can thus be written as

$$
\begin{aligned}
& q_{p, i}=e^{-\pi \lambda_{p} R_{e}^{2}}+\left(1-e^{-\pi \lambda_{p} R_{e}^{2}}\right) \times \operatorname{Pr}\left[R_{b}<P_{t h}\right] \\
& =e^{-\pi \lambda_{p} R_{e}^{2}}+\left(1-e^{-\pi \lambda_{p} R_{e}^{2}}\right)\left(1-e^{-\frac{P_{t h}}{P_{b}}}\left(\frac{1-e^{-\pi \lambda_{p}}}{1-e^{-\pi \lambda_{p} R_{e}^{2}}}\right)\right. \\
& \left.\quad-\int_{1}^{R_{e}} \frac{2 \pi \lambda_{p} r_{p, 1 \backslash i}}{1-e^{-\pi \lambda_{p} R_{e}^{2}}} e^{-\frac{P_{t h}}{P_{b} r_{p, 1 \backslash i}^{-\alpha}}} e^{-\pi \lambda_{p} r_{p, 1 \backslash i}^{2}} d r_{p, 1 \backslash i}\right),
\end{aligned}
$$

and the integration in (10) can be performed numerically.

\section{CO-OPERATIVE SPECTRUM SENSING}

We will now look at two specific co-operating schemes. The co-operating nodes indicate the channel occupancy sensed by them in the form of separate narrowband beacons. For each scheme, we will first evaluate the misdetection probability of a co-operating node's beacon, and then will evaluate the final misdetection probability taking into account the primary beacons and secondary node co-operations. It should be noted that we assume each co-operating node shares its sensed information regarding the primary beacons, and not the final decision obtained after co-operation.

\section{A. Nearest secondary node co-operation}

In nearest node co-operation, each secondary node $\phi_{s, i}$ first selects its closest neighbor $\phi_{s, 1 \backslash i} \in \Phi_{s}$. Because the signals from $\phi_{s, 1 \backslash i}$ with $r_{1}<R_{e}$ are neglected due to path loss, there may be an occasion where a $\phi_{s, 1 \backslash i}$ does not exist for cooperation. If this probability is $\rho_{0}, r h_{0}=e^{-\pi \lambda_{s} R_{e}^{2}}$. Let $r_{1 \backslash i}$ be the distance from $\phi_{s, i}$ to $\phi_{s, 1 \backslash i}$ whenever $r_{1}<R_{e}$. The PDF of $r_{1 \backslash i}$ is obtained as $f_{r_{1 \backslash i}}(x)=\frac{2 \pi \lambda_{s} x}{1-e^{-\pi \lambda_{s} R_{e}^{2}}} e^{-\pi \lambda_{s} x^{2}}, 0<$ $x<R_{e}$.

$\phi_{s, 1 \backslash i}$ senses the presence of primary receiver beacons, and passes that information in the form of binary information in a narrow band channel using another beacon signal. Let $P_{b, s}$ be the power of this beacon, and $\left|h_{i, 1 \backslash i}\right|^{2}$ be the channel power gain between $\phi_{s, i}$ and $\phi_{s, 1 \backslash i}$. Therefore, if the received beacon power $\left(P_{R, s}\right)$ at $\phi_{s, i}$ from $\phi_{s, 1 \backslash i}$ is given by $P_{R, s}=P_{b, s}\left|h_{i, 1 \backslash i}\right|^{2} g\left(r_{1 \backslash i}\right)$, where $g\left(r_{1 \backslash i}\right)$ is the path loss between $\phi_{s, i}$ and $\phi_{s, 1 \backslash i}$.

Let $q_{s, i}$ be the probability of misdetecting the co-operating node's beacon when $\phi_{s, 1 \backslash i}$ exists. It is obtained as

$$
q_{s, i}=\operatorname{Pr}\left[P_{R, s}<P_{t h}\right]=E_{r_{1 \backslash i}}\left[1-e^{-\frac{P_{t h}}{P_{b, s} g\left(r_{1 \backslash i}\right)}}\right] .
$$

The simplified expression for $q_{s, i}$ is

$$
\begin{aligned}
q_{s, i} & =1-e^{-\frac{P_{t h}}{P_{b, s}}}\left(\frac{1-e^{-\pi \lambda_{s}}}{1-e^{-\pi \lambda_{s} R_{e}^{2}}}\right) \\
& -\int_{1}^{R_{e}} \frac{2 \pi \lambda_{s} r_{1 \backslash i}}{1-e^{-\pi \lambda_{s} R_{e}^{2}}} e^{-\frac{P_{t h}}{P_{b} r_{1 \backslash i}^{-\alpha}}} e^{-\pi \lambda_{s} r_{1 \backslash i}^{2}} d r_{1 \backslash i} .
\end{aligned}
$$

Let $q_{1}$ be the final misdetection probability of $\phi_{s, i}$ when co-operating with its closest neighbor. We will assume that $\phi_{s, i}$ uses an OR rule [12] where $q_{1}$ becomes the product of the separate primary and secondary beacon misdetecting probabilities. However, the probability that $\phi_{s, 1 \backslash i}$ does not exist needs to be taken into account. $q_{1}$ is composed of the following events: (1) $\phi_{s, 1 \backslash i}$ does not exist, and $\phi_{s, i}$ misdetects, (2) $\phi_{s, 1 \backslash i}$ does exist, but both $\phi_{s, 1 \backslash i}$ and $\phi_{s, i}$ misdetect the primary beacons, and (3) $\phi_{s, 1 \backslash i}$ does exist, and detects the primary beacon correctly, but $\phi_{s, i}$ misdetects both the primary system beacons and the beacon from $\phi_{s, 1 \backslash i}$. Thus, we can write $q_{1}$ as

$q_{1}=q_{p, i}\left(e^{-\pi \lambda_{s} R_{e}^{2}}+\left(1-e^{-\pi \lambda_{s} R_{e}^{2}}\right)\left(q_{p, i}+\left(1-q_{p, i}\right) q_{s, i}\right)\right)$. 
We have used the fact that $q_{p, i}$ is the same $\forall i \in \Phi_{s}$. Furthermore, correct secondary beacon reception due to double errors ( $\phi_{s, 1 \backslash i}$ misdetects the primary beacons but $\phi_{s, i}$ detects a secondary beacon when it's not present) are negligible. Moreover, spatial correlations have not been taken into account in the derivation of (13).

B. Co-operating with $M$ secondary nodes within a given radius

In this scheme, $\phi_{s, i}$ would select $M$ secondary nodes for co-operation randomly within a co-operating radius of $R_{c}(<<$ $R_{e}$ ). If the number of other secondary nodes within $R_{c}$ is less than $M$, all the available nodes would be used for cooperation. We will assume that the selected nodes are always available for co-operation.

Let $\phi_{s, R_{c} \backslash i} \in \Phi_{s}$ be any secondary node within a distance of $R_{c}$ from $\phi_{s, i}$. It's location is denoted as $x_{R_{c} \backslash i} \in \mathbb{R}^{2}$, and the distance from $\phi_{s, i}$ to $\phi_{s, R_{c} \backslash i}$ is denoted as $r_{R_{c} 1} \cdot r_{R_{c} \backslash i}$ is written as $\left\|x_{R_{c} \backslash i}-x_{i}\right\|$. The distribution of $r_{R_{c} \backslash i}$ is obtained in a similar manner to (2) as $f_{r_{R_{c} \backslash i}}(x)=2 \frac{x}{R_{c}^{2}}, 0<x<R_{c}$, and 0 otherwise.

Similar to the nearest node co-operation, whenever a $\phi_{s, R_{c} \backslash i}$ detects the primary beacons, it would share the information using another beacon signal to $\phi_{s, i}$. We assume that $\phi_{s, i}$ can differentiate the beacons coming from the $N$ associated secondary nodes, which can be easily achieved via orthogonal codes serving as an identifier of each secondary node within $\Phi_{s}$. If $\left|h_{i, R_{c} \backslash i}\right|^{2}$ and $g\left(r_{R_{c} \backslash i}\right)$ are the channel gain and path loss between $\phi_{s, R_{c} \backslash i}$ and $\phi_{s, i}$, the received beacon power $P_{R, s}$ from $\phi_{s, R_{c} \backslash i}$ is given by $P_{R, s}=P_{b, s}\left|h_{i, R_{c} \backslash i}\right|^{2} g\left(r_{R_{c} \backslash i}\right)$.

If $q_{s, i, R_{c}}$ is the probability of $\phi_{s, i}$ misdetecting the beacon from $\phi_{s, R_{c} \backslash i}$, it is obtained as

$$
q_{s, i, R_{c}}=1-\frac{e^{-\frac{P_{t h}}{P_{b, s}}}}{R_{c}^{2}}-\frac{2}{R_{c}^{2}} \int_{1}^{R_{c}} e^{-\frac{P_{t h}}{P_{b, s} r_{R_{c} \backslash i}^{-\alpha}}} r_{R_{c} \backslash i} d r_{R_{c} \backslash i} .
$$

Let $q_{2}$ be the final misdetection probability of $\phi_{s, i}$ when co-operating with $M$ random secondary neighbors within a co-operating distance of $R_{c}$. We will again assume an OR rule at $\phi_{s, i}$ where it is enough to detect a single affirmation about the presence of primary spectrum occupation, and no spatial correlation. Although $M$ secondary nodes are ideally selected for co-operation, due to the random nature of $\Phi_{s}$, the number of co-operating nodes may be less than $M$ with finite probabilities. Thus, $q_{2}$ is the sum of several probability components corresponding to the number of co-operating nodes. Let $q$ be the probability of misdetection from arising from a single cooperating node (sum of the primary beacon misdetection probability by $\phi_{s, R_{c} \backslash i}$ and the probability that the beacon of $\phi_{s, R_{c} \backslash i}$ is misdetected by $\phi_{s, i}$ when $\phi_{s, R_{c} \backslash i}$ correctly detects the primary beacons). $q$ is written as $q=\left(q_{p, i}+\left(1-q_{p, i}\right) q_{s, i}\right)$. Whenever a given $k(\leq M)$ co-operating nodes are present, the final misdetecting probability of $\phi_{s, i}$ becomes $q_{p, i} q^{k}$. As such, $q_{2}=E_{k}\left[q_{p, i} q^{k}\right]$, where $0 \leq k \leq M$. After averaging and some mathematical modifications, $q_{2}$ becomes

$q_{2}=q_{p, i}\left(e^{-\pi \lambda_{s} R_{c}^{2}(1-q)} \frac{\Gamma\left(M, \pi \lambda_{s} R_{c}^{2} q\right)}{\Gamma(M)}+\left(1-\frac{\Gamma\left(M, \pi \lambda_{s} R_{c}^{2}\right)}{\Gamma(M)}\right) q^{M}\right)$

\section{PRIMARY SYSTEM PERFORMANCE}

We now look at the impact of the secondary node misdetections on the primary receiver outage. Let $\gamma_{p, j}$ be the received SINR at the $j$-th primary receiver $\phi_{p, j}, I$ be the aggregate interference from the secondary nodes which misdetect the beacon and transmit their signals within the primary user's spectrum, $P_{R, p, j}$ is the received primary signal power at $\phi_{p, j}$, and $\sigma_{n}^{2}$ be the noise power spectral density at a primary receiver. $P_{R, p, j}$ is written as $P_{R, p, j}=P_{p}\left|h_{p, j}\right|^{2} r_{p, j}^{-\alpha}$, where $P_{p},\left|h_{p, j}\right|$ and $r_{p, j}$ are respectively the primary transmit power, channel power gain and distance between the primary transmitter and $\phi_{p, j}$. We can thus write $\gamma_{p, j}$ as $\gamma_{p, j}=\frac{P_{R, p, j}}{I+\sigma_{n}^{2}}$. An outage is said to occur whenever $\gamma_{p, j}<\gamma_{t h}$ where $\gamma_{t h}$ is a threshold SINR value. Note that we are more interested in the SINR falling below a threshold for the primary signals as opposed to the received signal falling below a threshold used for beacon detection. The primary signals would be transmitting data whereas the beacon signals only indicate the channel occupation for which the received signal level was sufficient. Thus, the outage probability of $\gamma_{p, j}$ denoted as $P_{\text {Out }, j}$ is written as $P_{O u t, j},=\operatorname{Pr}\left[\gamma_{p, j}<\gamma_{t h}\right]$. This is obtained as $P_{\text {Out }, j}=1-e^{\left(-\frac{\gamma_{t h} \sigma_{n}^{2}}{P_{p} r_{p, j}^{-\alpha}}\right)} M_{I}\left(\frac{\gamma_{t h}}{P_{p} r_{p, j}^{-\alpha}}\right)$.

To evaluate this, the MGF of the aggregate interference at $\phi_{p, j}\left(M_{I}(s)\right)$ needs to be obtained. $M_{I}(s)$ is written as $M_{I}(s)=E\left[e^{-s I}\right]$. Let $r_{j, \nu}$ be the distance from $\phi_{p, j}$ to the $\nu$ th interfering secondary node $\phi_{s, \nu}$ distributed as (2). Note that similar to Section III, we do not consider the interference from $\phi_{s, \nu}$ whenever $r_{j, \nu}>R_{e}$. When $q \in\left\{q_{1}, q_{2}\right\}$ is the misdetection probability of $\phi_{s, i}$ after co-operative spectrum sensing, the Coloring theorem [15] can be used to obtain the intensity of the interfering secondary nodes distributed in $\mathbb{R}^{2}$ as $q \lambda_{s}$. $M_{I}(s)$ is thus obtained as $M_{I}(s)=e^{\pi R_{e}^{2} q \lambda_{s}\left(M_{I_{\nu}}(s)-1\right)}$, where $M_{I_{\nu}}(s)$ is the MGF of the interference from $\phi_{s, \nu} . M_{I_{\nu}}(s)$ is written as $M_{I_{\nu}}(s)=E\left[e^{-s P_{s}\left|h_{j, \nu}\right|^{2} g\left(r_{j, \nu}\right)}\right]$, where $P_{s}$ is the secondary signal transmit power, and $\left|h_{j, \nu}\right|^{2}$ and $g\left(r_{j, \nu}\right)$ are respectively the channel gain and the path loss between $\phi_{p, j}$ and $\phi_{s, \nu} \cdot M_{I_{\nu}}(s)$ is derived as

$$
M_{I_{\nu}}(s)=\frac{1}{R_{e}^{2}}\left(\sum_{k=0}^{\infty}\left(-s P_{s}\right)^{k}+\sum_{l=0}^{\infty} 2\left(-s P_{s}\right)^{l} \frac{R_{e}^{2-\alpha l}-1}{2-\alpha l}\right)
$$

We will now provide numerical results on the total misdetection probability with respect to the different co-operation and primary beacon detection schemes. Due to space constraints, we will not elaborate on the primary receiver outage. Parameter values of $R_{e}=1500, R_{c}=500, \alpha=3, P_{b, s}=-40 \mathrm{dBm}$, and $P_{b}=-50 \mathrm{dBm}$ will be used. $P_{b}$ has been set $10 \mathrm{~dB}$ lower than $P_{b, s}$ because the primary system's energy should not be used excessively to support the secondary nodes.

Fig. (1) plots the total misdetection probability with respect to the detection threshold of the secondary nodes $\left(P_{t h}\right)$. While co-operation does not significantly decrease the misdetection probability for higher $P_{t h}$, there is a marked deacrease when $P_{t h}$ is lower. When $P_{t h}=-120 \mathrm{dBm}$ and using $M$ cooperation, there can be as much as a $10^{4}$ fold decrease in 
the total misdetection probability when co-operation takes place. Separately detecting all the primary beacons performs better than sensing the beacon of the closest primary receiver. However, as mentioned before, this comes at the cost of additional complexity and resources. It is also interesting to note that while closest co-operation performs better than $M$ co-operation when $P_{t h}$ is higher while the converse is true for lower $P_{t h}$.

The behavior of the total misdetection probability for $M$ co-operation is investigated in Fig. (2) under different $M$ and $\lambda_{p}$. For both separate primary beacon detection and closest primary beacon detection, the misdetection probability approaches 1 when $\lambda_{p}$ is low. Increasing the number of co-operating nodes $M$ does not help significantly. However, when $\lambda_{p}$ increases to $10^{-3}$, increasing $M$ has some effect. Furthermore, the performance gap between separate primary beacon detection and closest primary beacon detection becomes apparent. Moreover, all curves flatten out indicating that the effect of $\lambda_{s}$ becomes negligible beyond $-40 \mathrm{~dB}$.

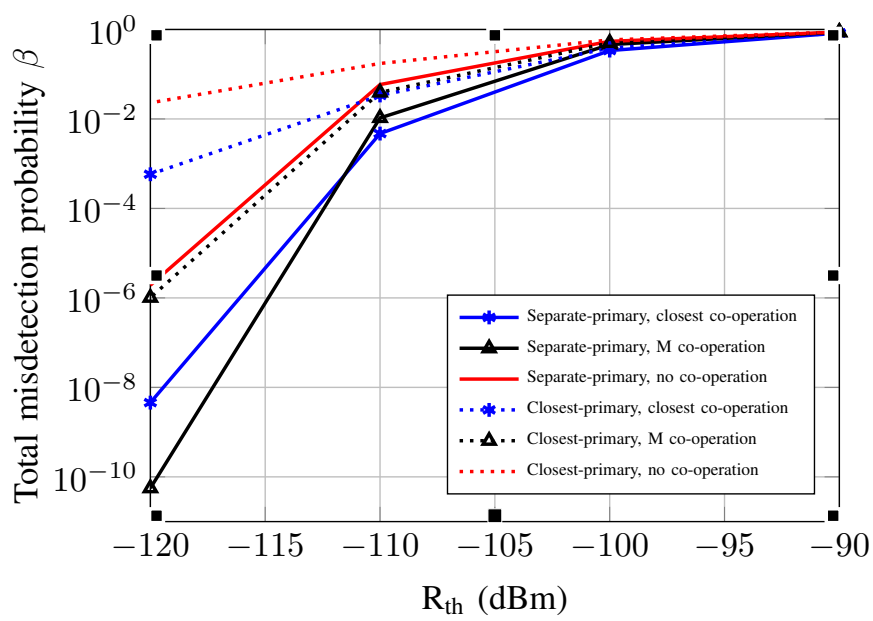

Fig. 1: Total misdetection probability vs. $P_{t h}$ for different cooperation schemes. $\lambda_{p}=0.0001, \lambda_{s}=0.0001$, and $M=10$.

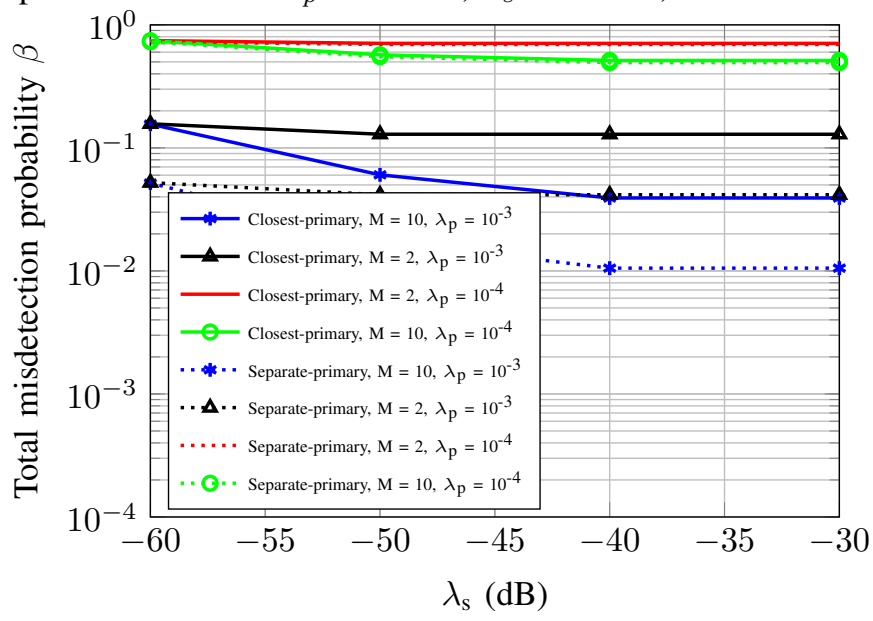

Fig. 2: Total misdetection probability vs. $\lambda_{s}$ for $M$ cooperation for different values of $M$ and $\lambda_{p} . P_{t h}=-110 \mathrm{dBm}$. VII. CONCLUSION

This paper investigated the total misdetection probability of a secondary node in an interweave CR network under different co-operation and primary beacon reception strategies. Two independent PPPs were considered for the primary receivers and secondary nodes along with independent channels with exponential path loss and Rayleigh fading. It was seen that $M$ cooperation performs better when the reception threshold $P_{t h}$ is lower, and that it provides a $10^{4}$ fold decrease in misdetection for $P_{t h}-120 \mathrm{dBm}$. Moreover, having a lower primary receiver density is detrimental irrespective of the co-operation scheme used. Future research directions include extending the work to include spatial and temporal correlation, and investigating the energy efficiency of co-operation schemes for CR networks.

\section{REFERENCES}

[1] I. F. Akyildiz, W.-Y. Lee, M. C. Vuran, and S. Mohanty, "Next generation/dynamic spectrum access/cognitive radio wireless networks: A survey," Computer Networks, vol. 50, no. 13, pp. 2127-2159, 2006. [Online]. Available: http://www.sciencedirect.com/science/article/pii/S1389128606001009

[2] A. Ghasemi and E. Sousa, "Interference aggregation in spectrum-sensing cognitive wireless networks," IEEE J. Sel. Areas Commun., vol. 2, no. 1 , pp. $41-56$, Feb. 2008

[3] H. Venkataraman and G. Muntean, Cognitive Radio and its Application for Next Generation Cellular and Wireless Networks. Springer, 2012.

[4] S. Atapattu, C. Tellambura, and H. Jiang, "Energy detection based cooperative spectrum sensing in cognitive radio networks," IEEE Trans. Wireless Commun., vol. 10, no. 4, pp. 1232-1241, April 2011.

[5] S. Mishra, A. Sahai, and R. Brodersen, "Cooperative sensing among cognitive radios," in Proc. IEEE ICC, vol. 4, June 2006, pp. 1658-1663.

[6] A. Ghasemi and E. Sousa, "Collaborative spectrum sensing for opportunistic access in fading environments," in Proc. IEEE DySPAN, Nov 2005, pp. 131-136.

[7] A. Rabbachin, T. Q. S. Quek, H. Shin, and M. Z. Win, "Cognitive network interference," IEEE J. Sel. Areas Commun., vol. 29, no. 2, pp. 480-493, Feb. 2011.

[8] P. Madhusudhanan, Y. Liu, and T. Brown, "On primary user coverage probabilities and faulty cognitive radios," IEEE Trans. Wireless. Commun., vol. 13, no. 11, pp. 6207-6218, Nov 2014.

[9] A. Babaei, P. Agrawal, and B. Jabbari, "Statistics of aggregate interference in cognitive wireless ad hoc networks," in Proc. IEEE ICNC, Jan 2012, pp. 397-401.

[10] Y. Sun and B. Mark, "Interference model for spectrum sensing with power control," in Proc. IEEE CISS, March 2013, pp. 1-6.

[11] S.-C. Hung and K.-C. Chen, "Geometric design of cooperative spectrum sensing for cognitive radios," in Proc. IEEE PIMRC, Sept 2013, pp. 2496-2501.

[12] M. Derakhshani and T. Le-Ngoc, "Aggregate interference and capacityoutage analysis in a cognitive radio network," IEEE Trans. Veh. Technol., vol. 61 , no. 1 , pp. 196-207, Jan. 2012.

[13] A. Busson, B. Jabbari, A. Babaei, and V. Veque, "Interference and throughput in spectrum sensing cognitive radio networks using point processes," Journal of Communications and Networks, vol. 16, no. 1, pp. 67-80, Feb 2014.

[14] M. Di Renzo, F. Graziosi, and F. Santucci, "Cooperative spectrum sensing in cognitive radio networks over correlated log-normal shadowing," in Proc. IEEE VTC, April 2009, pp. 1-5.

[15] J. F. Kingman, Poisson Processes. Oxford University Press, 1993.

[16] I. Gradshteyn and I. Ryzhik, Table of integrals, Series, and Products, 7th ed. Academic Press, 2007.

[17] A. Baddeley, I. Barany, R. Schneider, and W. Weil, Spatial Point Processes and their Applications. Springer, 2007.

[18] A. Goldsmith, Wireless Communications. Cambridge University Press, 2005.

[19] L. Barlemann and S. Mangold, Cognitive Radio and Dynamic Spectrum Access. Wiley, 2009.

[20] D. Moltchanov, "Distance distributions in random networks," Ad Hoc Networks, vol. 10, no. 6, pp. 1146-1166, 2012. [Online]. Available: http://www.sciencedirect.com/science/article/pii/S1570870512000224

[21] D. Stoyan, W. S. Stoyan, Kendall, and J. Mecke, Stochastic Geometry and it's Applications. John Wiley \& Sons Ltd., 1995. 